\title{
Growth and remodeling of the human maxilla
}

\author{
DONALDH. ENLOW, PH.T., and SFONG BANG, D.D.S. \\ Ann Arbor, Mich.
}

THE purpose of the present study is to describe and interpret the sequence of remodeling changes which take place during the postnatal growth of the young human maxilla. This report is a sequel to previous studies dealing with principles of bone remodeling and with the postnatal growth of the human mandible..$^{1-4}$ As the growing maxilla increases in size, there is a companion process of remodeling which functions to maintain the constant proportions of the bone's shape and the relative positions of its component parts. This process of remodeling involves sequential, localized adjustments in bone structure during the constant changes produced by the growth of the entire maxilla. An understanding of these remodeling changes is basic to a meaningful interpretation of the over-all process of growth.

\section{REVIEW OF THE LITERATURE}

Present knowledge of normal maxillary structure and growth is the result of many diverse studies representing a variety of biologic areas and methods, including comparative anatomy and paleontology, ${ }^{5,6}$ anthropology and anthropometry, ${ }^{7, s}$ embryology, ${ }^{9-11}$ vital staining, ${ }^{12,15}$ cephalometries, ${ }^{16-19}$ and experimental biology. ${ }^{20,21}$ Summaries of such information from varied points of view are found in standard texts and references. ${ }^{22-25}$

The pioneer works of John Hunter established the basic concept that bone growth proceeds by a process of outer surface deposition in conjunction with inner surface resorption. The vital-staining studies of $\mathrm{Brash}^{13,14}$ led to the belief that cranial growth proceeds largely by this same mechanism of periosteal bone deposition with proportionate endosteal removal. It was also held that adjustments in structure occur at sutures in order to maintain functional contact between adjacent bones. However, Massler and Schour ${ }^{26}$ have suggested that the sutures themselves represent actual primary centers of growth. The

From the Department of Anatomy, University of Michigan. 
influence of associated soft-tissue expansion upon such sutural growth has since been investigated, and it has been proposed that the growing brain, tongue, and eve all produce a continual separation of overlying sutures which stimulates bone growth at their free margins. Detailed histologic and histogenetic studies of connective tissue structure and organization within such growing sutures have also been made. ${ }^{21,27,} 28$ The various interstitially growing cartilages of the developing braincase have been suggested as primary growth stimulants during osteogenesis. During facial growth, the cartilaginous nasal septum is believed to be a primary force in pacing morphogenesis of the maxilla and other adjacent bones. ${ }^{29,}{ }^{30}$ The forward and downward growth of this septum would serve to "carry" the maxilla with it, thus tending to separate its various sutures and to stimulate new bone deposition on these same sutural surfaces. Relative timing has been found to be a factor in the comparative dominance of suture growth, either within the suture itself or on a nearby surface, versus generalized surface deposition of new bone. ${ }^{20}$ It has been estimated that growth by suture activity combined with cartilage expansion ceases after about the seventh postnatal year and that over-all surface apposition then comes to represent the dominant mechanism of growth. ${ }^{31}$ During periods of sutural growth, the particular sutures that are believed to contribute predominantly to maxillary growth are those articulating with the frontal, zygomatic, ethmoid, and palatine bones. ${ }^{23}, 31$ It is currently held that the maxillary bone is thrust in a downward and forward direction by growth at its articular and posterior margins. This conclusion is based on sutural orientation in addition to vital-staining experiments and serial cephalometric studies. ${ }^{16,21}$

\section{MATERIALS AND METHODS}

The complete right halves of the maxillary bones from twelve well-preserved human skulls, all with either deciduous or mixed dentitions, were serially sectioned and studied. The left side of each maxilla was left intact on the skull for section orientation and for mapping of the detailed distribution of the various kinds of bone deposits. Because of the architectural complexity as well as the fragility of the maxilla, tissue preparation by thin-section grinding and polishing methods could not be effectively employed. Sections were therefore prepared by routine methods of decalcification and microtome sectioning.

Because of the complex shape and contours of the maxilla, the entire bone was cut into several individual blocks in order to make possible transverse or longitudinal planes of section through each of the various areas. Frontal sections were avoided, since they do not provide a complete cut through the entire cortical plate. Each block of dried bone was decalcified and embedded in paraffin by ordinary techniques, and then serially sectioned at 15 microns. The serial sections were mounted in sequence on 2 by 3 inch glass slides, stained with hematoxylin and eosin, and covered. A code system was used for identification of blocks and location of individual sections within blocks. Composite pictures representing the characteristic and most commonly observed patterns of structure were then made (Fig. $1, C$ and $D$ ). The incidence of significant variations was also determined, described, and evaluated. 


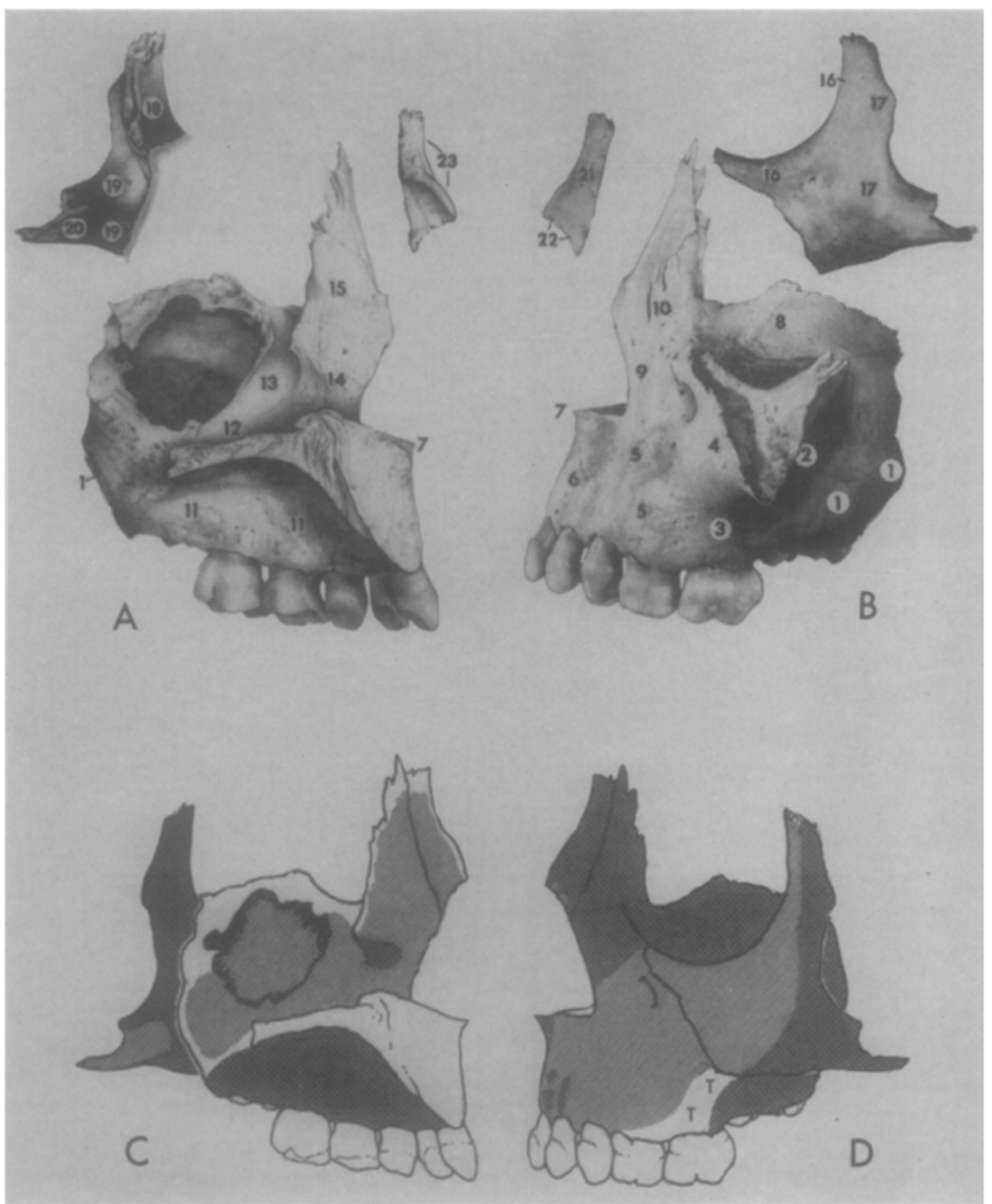

Fig. 1. $A$ and $B$, Diagrams indicating distribution of the various numbered areas deseribed in the text. $A$ shows a medial view of the maxilla, and $B$ shows a lateral view. Inset figures show lateral and medial views of the nasal and zygomatic bones. $C$ and $D$, Diagrams of medial (C) and lateral (D) aspects of the maxilla summarizing distribution of various periosteal (dark crosshatch) and endosteal (light crosshatch) bone deposits. Note zone of variable endosteal-periosteal transition $(T)$ on lateral cortex in molar area. For detailed discussion of each area in all parts of the maxillary bone, see text.

The technique of mapping the localized distribution of the various kinds of endosteal and periosteal bone deposits in all areas of a bone permits detailed analysis of the specific directions of growth in each part of the bone. The method also makes possible an understanding of the sequence of remodeling changes which accompany these growth movements. Such structural and growth analyses are based on sevcral principles of bone growth and remodeling. These include 
the concept of "area relocation," the "principle of the $V$," and the simple but fundamental principle that the particular surfaces which face the actual direetion of growth are those which receive new deposits of bone. Contralateral surfaces which face away from the direction of growth are typically resorptive. Such surfaces of resorption may be either endosteal or periosteal in nature and occur in all locations where the cortex is growing or drifting in corresponding cndosteal or periosteal dircetions. Detailed diseussions of these principles and deseriptions of the various kinds of periosteal and endosteal bone tissues have been presented in previous studies. ${ }^{1-4}$ In the present study, the regional distribution of specific periosteal and endosteal tissue deposits in all areas of the growing maxillary, nasal, and zygomatic bones is described and discussed. Using these observations as a basis, we will explain and evaluate the varions directions of growth movements.

\section{OBSERVATIONS}

Reference to Figs. 1 and 10 shows that the different parts of the maxilla are growing and moving in a complex variety of mesial (anterior), distal (posterior), superior, inferior, medial (palatal or lingual), and lateral (buceal or labial) directions. The over-all plan of maxillary growth is much more complex than the simplified generalization stating that the maxilla grows "downward and forward by a process of bone deposition on posterior and superior surfaces." Actually, the various parts of the maxilla are growing in a wide variety of individual directions. The above generalization also does not consider the important and key process of structural remodeling which necessarily accompanies growth. A study of the latter process serves to elarify the developmental basis for the series of specific and complex growth movements which oceur as the maxilla increases in over-all size.

THE MAXILLARY TUBEROSITY. During growth, the entire maxilla is moved and repositioned in a progressive anterior direction. As it does so, new bone deposits are simultaneonsly added onto the periosteal surfaces of the maxillary tuberosity (area 1 in Fig. 1, $A$ ). These additions increase the longitudinal dimensions of the growing maxilla and, at the same time, function to lengthen the dental arch as the teeth increase in number. During the active period of maxillary growth, deposits of bone on these posterior-facing surfaces are typically in the form of fine cancellous, nonlamellar bone tissue which later receives lamellar compaction. This type of bone tissue characteristically develops in skeletal areas involving particularly rapid growth. ${ }^{3}$ The presence of a relatively thick cortex composed of such bone tissue reflects the active contribution of this area to general maxillary growth. Since this surface faces posteriorly and somewhat laterally, apposition of new bone on its periosteal surface brings about growth in corresponding posterior and lateral directions. This produces a lengthening and a slight widening of the maxillary arch at its posterior end (Figs. 2, A and $10, B)$.

THE ZYGOMATIC PROCESS OF THE MAXILla. The posterior surface of the zygomatic process (area 2) receives new deposits of bone during maxillary growth. Its cortex is composed entirely of periosteal bone tissue, and the endosteal sur- 

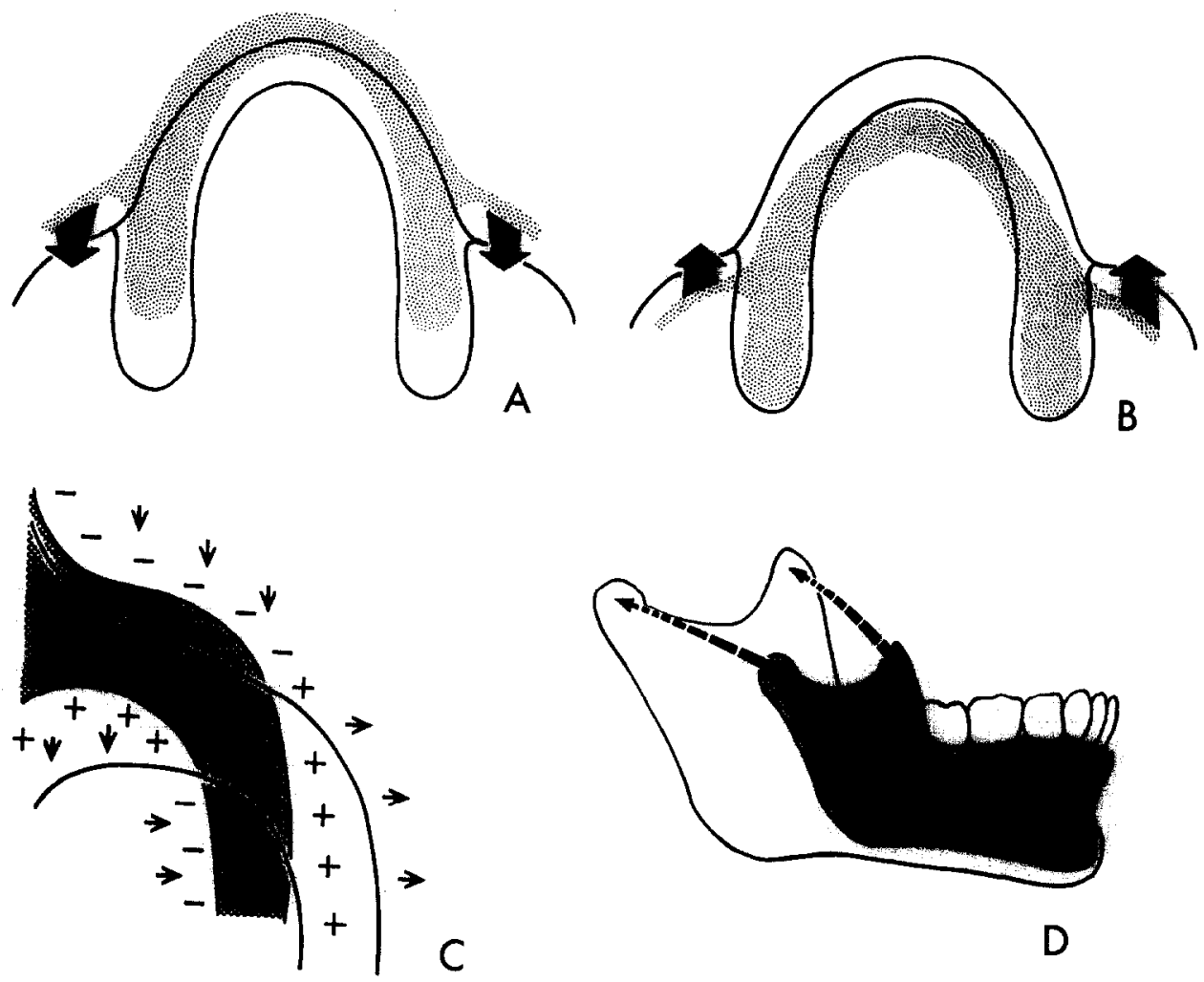

Fig. 2. A, Schematic interpretation of posterior growth of the maxillary arch and the zygomatic processes. Bone growth proceeds along the entire inner (lingual) side of the arch as well as along its posterior margin (maxillary tuberosity) and the posterior face of the zygomatic process. Resorptive removal occurs from the outer cortex of the premaxillary area and from the anterior surface of the zygomatic process. $B$ schematizes the apparent direction of growth which results from the anterior thrust of the maxillary body accompanying its actual growth in a posterior direction. $C$, Schematic diagram illustrating the mechanism of posterior and lateral movement of the combined zygomatic process of the maxilla and the adjacent zygomatic bone. Bone deposition (+) proceeds in lateral and posterior directions (arrows), together with complementary resorption (-) from anterior and medial surfaces. $D$, The generalized mode of maxillary growth and remodeling parallels that of the human mandible, shown here for comparison. During posterior growth of the condyle and ramus, the coronoid process is continuously relocated in a posterior direction (arrows). Similarly, the zygomatic process of the maxilla also receives proportionate posterior relocation as the maxillary body grows in this direction.

face is resorptive in nature. This area moves in a posterior course as the maxillary arch itself increases in longitudinal dimensions (Fig. 2, C).

In contrast to this pattern of the posterior surface, the facial (anterior) side of the zygomatic process (area 4 ) and the anterior-facing periosteal surfaces of area 5 are resorptive in character. The cortex is composed of endosteal bone which was formed by deposition on the inner or endosteal surface with corresponding removal from the outer or periosteal cortical surface. These areas 
of the face move in an actual posterior direction toward the base of the skull as the entire maxillary bone increases in size (Fig. 10, B). Without an interpretation of the remodeling basis for this particular movement, such growth activity would seem to contradict the principle that the maxilla moves "forward and downward." It is paradoxical that the maxilla grows in a forward direction; yet this forward-facing surface is actually resorptive in nature.

The maxilla does not grow forward by a single process of adding new bone on its various anterior surfaces. Rather, the entire maxillary bone becomes repositioned in this direction as simultaneous deposition of new bone occurs along posterior surfaces (Fig. 2, A). This situation is comparable to the growth sequence of the mandible. Mandibular growth takes place in the posterior manner indicated in Fig. 2, $D$, but the entire mandible is continuously moved in a forward direction. Similarly, the apparent development of the maxillary bone is also forward, but actual growth additions in areas 1 and 2 proceed backward (posteriorly).

The key point to an interpretation of the developmental basis for the resorptive nature of the anterior-facing maxillary surfaces in the malar region of the face lies in the fundamental remodeling principle of "area relocation." As new bone deposits are added onto posterior surfaces of the maxillary body, the anterior-posterior dimensions of the maxilla increase. However, constant relative positions of the various parts of the maxilla must be maintained during these increases. Simple, progressive additions of new bone on posterior surfaces without corresponding shifts of all pre-existing areas would result in progressively disproportionate relationships between the various new and old parts of the maxilla. The entire anterior surface of the zygomatic process of the maxilla and the facial surface of the maxillary body are, therefore, relocated in a continual posterior direction in order to maintain their constant relative positions in the growing maxilla as a whole. This is accomplished by a combination of bone deposition on the posterior surfaces of the zygomatic processes (area 2) with resorption from its anterior face (area 4). Thus, as the maxilla lengthens by new growth on its posterior margin, the zygomatic process moves continuously and simultaneously in a corresponding direction by a process of relocation. This particular remodeling movement can be compared with a parallel situation found in the growing mandible. Just as the coronoid process of the mandible becomes relocated in a continual posterior direction during the posterior growth of the ramus (Fig. 2, $D$, arrows), the zygomatic process of the maxilla also becomes relocated in a corresponding posterior direction as it keeps pace with the posterior growth of the maxillary body.

Microscopic sections of the cortex from areas 4 and 5 are composed of typical endosteal bone tissues. This cortical bone was formed by apposition on the inner or endosteal surface of the inward-moving compacta together with resorptive removal from the outer periosteal bone surface. In those parts of the maxilla where such inward growth has proceeded into regions which did not contain cancellous trabeculae, the resulting cortex is composed entirely of uniformly arranged endosteal circumferential lamellae. On the other hand, if this inward growth had taken place in medullary areas which already contained cancellous 
trabeculae, the resulting cortex is made of irregular, convoluted patterns of compacted cancellous bone tissue" (as in Fig. 6).

The orbital side of the frontal process (area 18) and the anterior face of the separate zygomatic bone (area 16) are resorptive in nature and, like the \%ygomatic process of the maxilla, grow and move in a general posterior course. The lateral rim of the orbit is also resorptive and grows posteriorly (Fig. 6). The posterior-facing surface of the zygomatic bone located within the temporal fossa (area 19) receives appositional bone deposits. Thus, the zygomatic bone is shifted in a backward direction corresponding to the posterior growth of the maxillary tuberosity and the zygomatic processes. It is successively relocated in position in order to maintain its relative location in the entire facial complex. During these growth and remodeling movements, the entire zygomatic arch is also increasing in general size by bone deposition on the lateral surface of the zygomatic arch (area $1 \tilde{r}$ ) with complementary resorption from the medial side

Fig. 3. The periosteal surface on the entire oral side (a) of the palatal process has received massive deposits of lamellar bone. Numerous anchoring fibers are seen in the cortex and were attached to overlying soft tissues. Note the formation of cancellous spaces in the middle areas of this periosteal bone. Such spaces follow the movement of the cortex in an oral dircetion, and former spaces became filled with bone on the nasal side of the palate (bottom of figure) as this side also moved in an oral course. This bone tissue is characteristic of compacted cancellous bone and has an irregular, convoluted structure.

Fig. 4. The lateral surface of the nose (frontal process of the maxilla) has a cortex composed of periosteal bone tissue (a). The opposite mucosal (inner) side is resorptive (b). The periosteal-endosteal contact is located on the crest of the rim at the anterior margin of this frontal process. The movements produced by the combination seen here cause growth of the nasal wall in the lateral, superior, and anterior directions which this surface faces.

Fig. 5. The mechanism of movement of a large medullary space. The area shown is a part of the frontal process of the maxilla. Sides $a$ and $c$ are depository, and surfaces $b$ and $d$ are resorptive. As the periosteal surfaces $a$ and $d$ move in a periosteal direction, the larger enclosed spaces, as seen here, move in the same manner hy a process of removal from the side toward the movement and corresponding deposition on the opposite surface.

Fig. 6. Photomicrograph showing the rim of the orbit as it passes over onto the surface of the lateral wall of the orbital cavity. Surface $a$ is the inner, endosteal surface and lias received deposits of bone. The outer, periosteal surface (b) is resorptive. The entire cortex is composed of endosteal bone and was moving in an endosteal direction.

Fig. 7 . The cortex of the premaxilla is composed of a thin plate of endosteal bone, as seen in this section taken from a site where the premaxillary surface is passing over onto the floor of the nasal cavity, which is also resorptive. Surface $a$ is endosteal and was receiving appositional deposits of bone. The outer periosteal surface $(b)$ is resorptive. The entire cortex was moving in an endosteal direction toward side $a$.

Fig. 8. The flour of the orbit is composed of a very thin cortex composed entirely of periesteal bone. Surface $a$ is periosteal, and growth by subperiosteal bone deposition has proceeded toward this side. The opposite endosteal surface (b), adjacent to the maxillary sinus, is resorptive. 
(area 20). This combination serves to widen the entire zygomatic arch simultaneously as it continues to move in its posterior course (Fig. 2, C).

THE MAXILLARY ARCH. The dimensions of the dental arch of the maxillary bone increase in the manner shown in Fig. 2, $A$. Note that the dominant area of growth occurs at the posterior margin of the arch on the maxillary tuberosity. Accompanying this, bone deposition takes place along the entire inner side of the maxillary arch together with contralateral resorption from the labial and

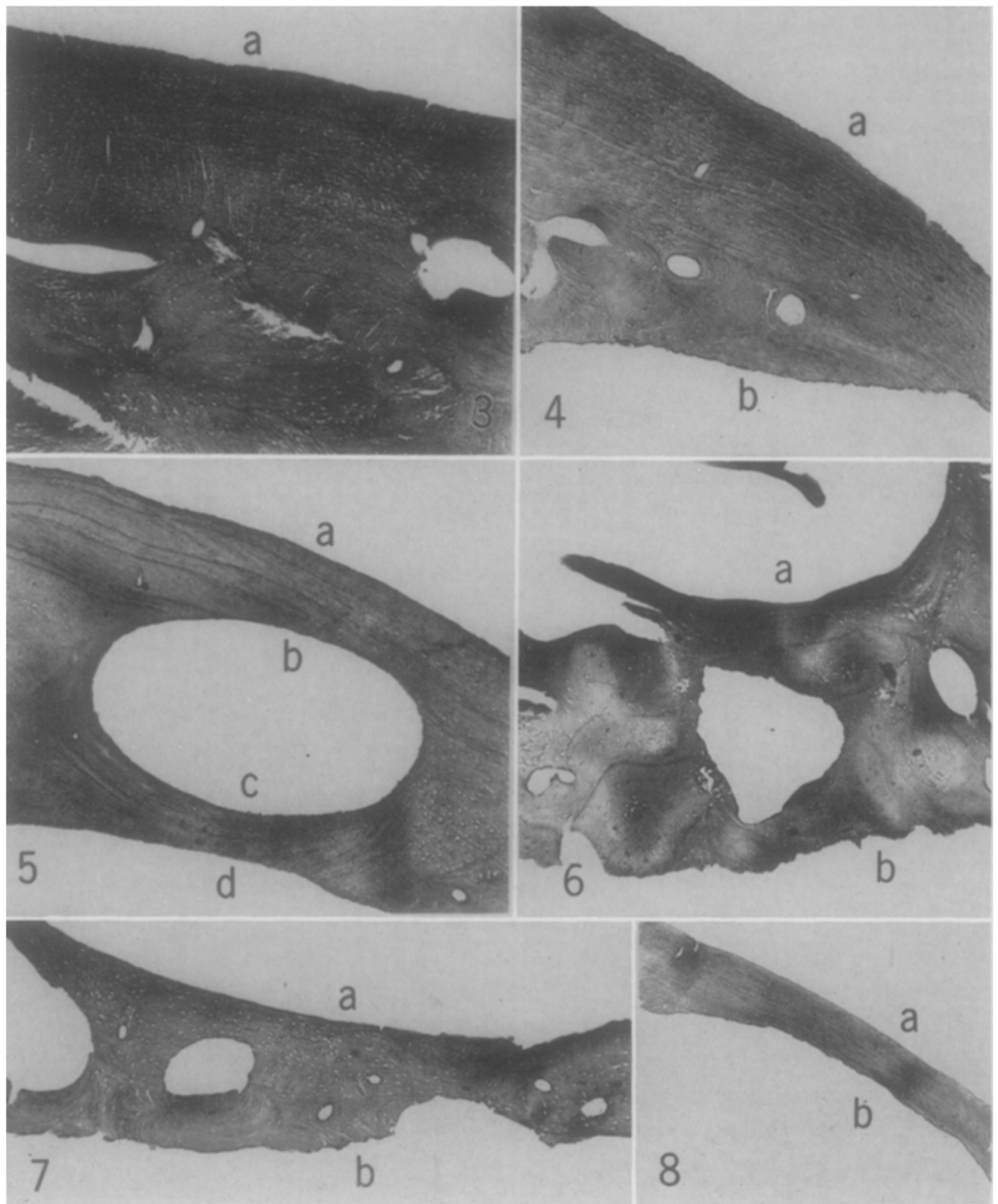

Figs. 3-8. For legends, see opposite page. 


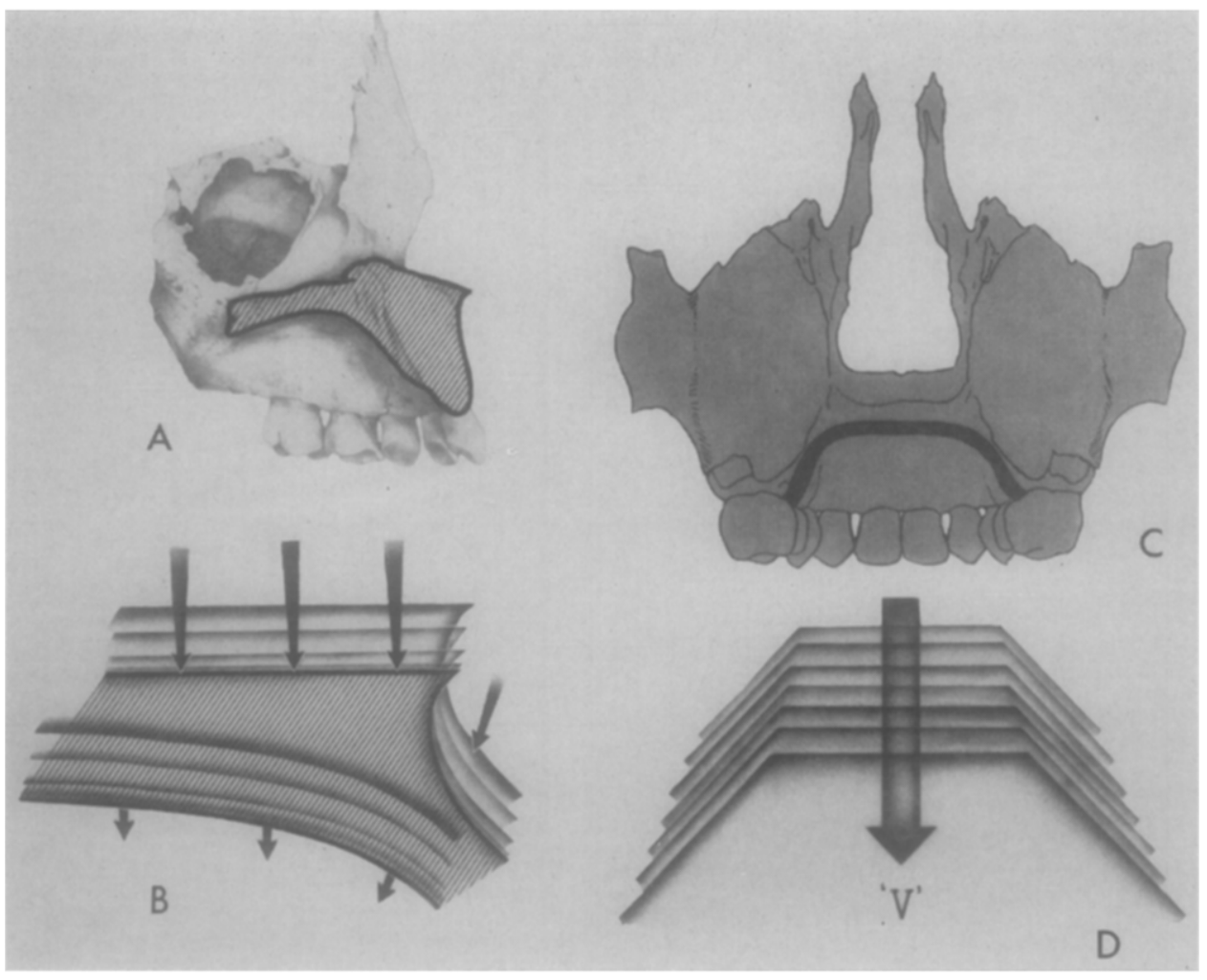

Fig. 9. $A$ and $B$, The oral palate (and floor of the nasal cavity) and the nasal spine move in a downward direction by bone deposition on the various inferior surfaces together with resorption from contralateral superior surfaces. The premaxillary area simultaneously moves downward and somewhat posteriorly by an equivalent process. $C$ and $D$, Following the "principle of the $v$ ", the palate grows in an inferior direction by subperiosteal bone deposition on its entire oral surface with corresponding resorptive removal from opposite surfaces. The entire $V$-shaped structure thereby moves in a direction toward the wide end of the $\checkmark$ and simultaneously increases in over-all size.

buccal sides in areas 5 and 6 . This growth sequence follows the $V$ principle in which a $V$-shaped structure (here, the dental arch itself) increases in size and at the same time moves in a direction toward the wide end of the $V$ by a combined process of apposition on the inner surface of the $V$ and resorption from outer surface. This combination of resorption from the labial side and deposition on the lingual surface of the arch also serves to shift the premaxillary area toward the midline. Since this area is growing in a posterior direction, it comes to lie in successive new locations which were formerly wider in their lateral dimensions. The lingual or medial direction of growth functions to decrease this width during such posterior relocation and thereby continuously maintains constant size proportions of the premaxilla. This is an example of "area relocation" as well as the "principle of the $V$ ", as described in previous studies.

As the maxilla grows in a general posterior direction, the entire bone is continuously "thrust" in an anterior course, as previously mentioned. Note, 


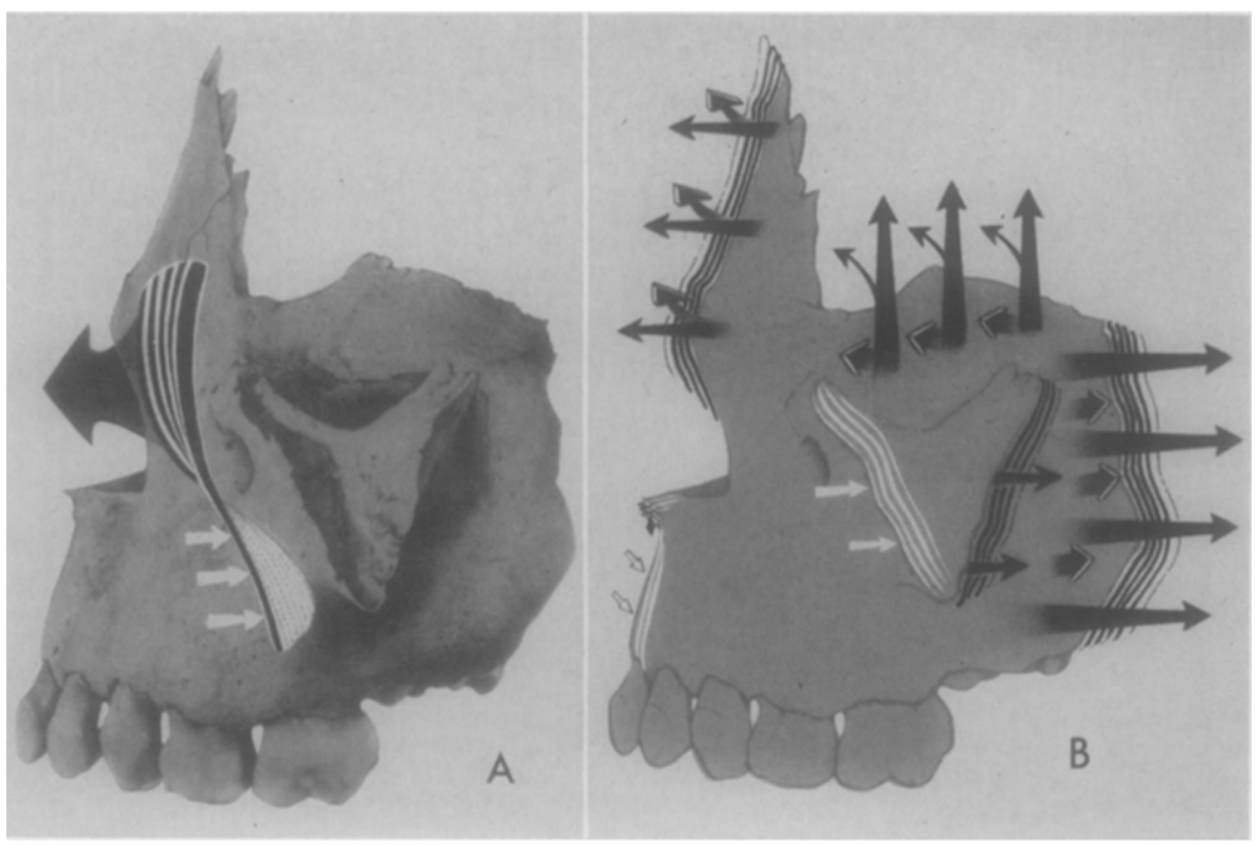

Fig. 10. A, Diagram showing the nature of growth along the complex curvature at the contact between the malar area and the lateral wall of the nasal region. This contour becomes expanded as the nasal area (frontal process of the maxilla and adjacent nasal bone) moves in progressive superior, lateral, and anterior directions (black arrow). Simultaneously, the malar area of the maxilla is receding in a posterior direction (white arrows). $B$, A diagrammatic, summary illustration showing the complex variety of remodeling movements which occur in the various parts of the maxilla as the entire bone grows in over-all size. See text for detailed description.

in Fig. 2, $A$ and $B$, that the zygomatic process of the maxilla is similarly being carried in an anterior course as it grows in an actual posterior direction.

The width of the dental arch increases progressively and proportionately in size by continued bone deposition along the lateral (buccal) surface of the maxillary tuberosity in the molar area posterior to the zygomatic process (area 1). This surface contour is so oriented that it faces in two general directionsposterior and lateral-and surface bone additions serve to bring about increased growth in corresponding posterior and lateral directions.

THE PALATINE PROCESSES OF THE MAXILLA. In conjunction with the various growth movements just deseribed, a complementary serics of changes occurs during the growth of the right and left palatine processes (areas 11 and 12). Fig. 9, $C$ shows that the sequential mechanism of size increase in this area conforms to the same basic principle of the $V$. As previously noted, the dental arch itself is a $V$-shaped structure which grows by deposition on the inside (lingual) surface together with resorption from some parts of the outer (labial) surface of this horizontally oriented $V$. The palatal arch shows a similar pattern of growth as new bone deposits are added on the oral surface with corresponding proportionate removal from the opposite nasal surface (Fig. 9, B). The vertically 
oriented $V$ of the palatal areh thus increases in size and undergoes a downward movement in conjunction with the growth of the entire maxillary arch as it grows in posterior and lateral directions. The inner side of this $V$ is represented by the oral surface of the palatal shelf. The outer side is the nasal surface of the palatine process and, in addition, the entire labial cortex of the maxilla and premaxilla anterior to the right and left premolars.

The palatal shelf of the maxilla varies regionally from a relatively thin sheet of simple lamellar bone to a rather thick plate composed of two cortical tables enclosing a middle cancellous diploë. In the former type of structure, the thin sheet of palatal bone receives uniform lamellar deposition on its oral side in combination with resorptive removal from the contralateral nasal side. It is evident that the bone tissue on the nasal surface of the palate is actually of oral origin but has become translocated by a shift over to the nasal side during progressive movement in an oral direction. Palatal areas involving a much thicker shelf than the single cortical plate just described grow and move in an oral direction by a combined process of periosteal apposition and endosteal resorption on the oral lamina together with endosteal apposition and periosteal resorption on the nasal lamina (Fig. 3). Since endosteal apposition on the nasal lamina occurs by a process of lamellar deposition within spongy diploë, its cortex is composed of irregular, convoluted, compact bone."

THE PREMAXILla. The labial surface of the cortex in the premaxillary region (area 6 ) is resorptive in character. The endosteal surface of this outer cortex receives appositional deposits so that the cortex of the premaxilla, like the maxillary cortices in adjacent areas 4 and 5 , is composed of endosteal bone tissue (Fig. 7). Cancellous compaction is involved in interdental areas during this endosteal direction of growth, and the resulting cortex is composed of typical irregular, convoluted bone tissues produced by lamellar deposition within canccllous spaces. 'T'he alveolar bone covering teeth, however, is relatively thin and is usually composed of a single plate of endosteal lamellar bone with a resorptive periosteal surface. Scattered, very thin patches of overlying periosteal bone have been observed in some of the specimens studied and may be a result of cortical adjustments to tooth movements occurring during eruption and drift. The lingual side of the cortex in the premaxillary area possesses a periosteal surface of deposition which continues directly into the periosteal bone of the palatal process.

The combination of resorption from the labial surface with deposition on the lingual side of the premaxilla brings about a growth movement which proceeds in a predominantly downward but at the same time slightly postcrior direction. This sequence is schematized in Fig. 9, B. (Compare also with Fig. 2, $A$.) Note that the alveolar process is elongating and increasing in depth by new bone additions at its free margin, so that a progressive extension of premaxillary alveolar bone occurs as it is simultaneously moving down and also drifting slightly posteriorly. The nasal spine moves in a corresponding downward direction by bone deposition on its inferior periosteal surface with contralateral surface removal from the nasal side. The principle of "area relocation" is involved in these various movements. In Fig. 9, $B$ note that as the premaxilla moves 
downward and somewhat posteriorly, regions that were formerly located at or near the margin of alveolar processes become successively relocated in their actual positions into the maxillary body or into the downward-moving nasal spine. As seen in this diagram, the process of relocation in this area is accomplished by periosteal resorption and endosteal apposition on the labial cortex, together with the converse combination of periosteal deposition and endosteal removal at the lingual cortex.

It will be noted in Figs. 1, $A, 9, B$, and 10 that the particular surfaces of the premaxillary area which face down are the endosteal side of the labial cortex and the periosteal side of the lingual cortex. Since the depth of the premaxilla can increase only by downward growth, it is apparent that the surfaces which actually face this direction are those which receive new bone deposits. Thus, the endosteal nature of premaxillary growth, involving periosteal resorption and endosteal apposition on the labial cortex, provides this downward growth since its endosteal surface faces this direction. It should be noted that the resorptive nature of the outer premaxillary cortex seems to be associated primarily with such a downward growth movement but that a degree of cortical recession in a posterior direction is also necessarily involved. The actual degree of recession is not now known and has not yet been determined by quantitative studies. It should be pointed out also that these various premaxillary growth patterns occur in man but that growth patterns in other species have not been studied and may be quite different. This is known to be true with respect to the mandible.

In cephalometric studies by Brodie, ${ }^{32}$ it was suggested that tecth in the incisor region can experience a "relative dropping back" with continued growth. Observations described in the preceding paragraphs would indicate that such dropping back represents an actual rather than a relative growth movement. It will be recalled that a similar growth process involving facial regression also oceurs during the postnatal growth of the human mandible. ${ }^{4,34}$ The incisor region of the mandible moves in a lingual direction by a combined process of periosteal resorption from the labial side and periosteal apposition on the lingual side. It has also been shown, however, that such cortical recession in the incisor region does not occur in the mandible of the rhesus monkey. ${ }^{3}$ The occurrence of an extensive resorptive cortex in an anterior region of the human maxilla, as well as the mandible, suggests a developmental and structural basis for the decreased prognathism characteristic of the human being in comparison with other primates. It is possible that the upright posture of man, together with his upright face, enlarged cranial cavity, vertically oriented nose, lack of protruding and nasal-obstructing jaws, and the occurrence of a recessive incisor region in both the mandible and the maxilla are all functionally interrelated. Adequate and detailed comparative histogenic and histologic information concerning both the upper and lower jawbones in a variety of species is not now available. The undertaking of such a study is encouraged.

THE ORBIT. The orbital surface of the maxillary bone (area 8 ) faces in three general directions : laterally, anteriorly, and superiorly. Deposition of bone on this surface brings about a size increase and a corresponding growth movement 
in each of these directions. The medial walls of the right and left orbits move farther apart during growth, thereby increasing the breadth of the nasal bridge and the underlying nasal cavities. At the same time, the anterior-facing slope of this same surface will produce a very slight growth movement in a rostral direction, thus contributing to the complex forward thrust of the posteriorgrowing maxillary body. A degree of vertical or superior movement is also involved, so that the general course of growth in combined lateral, anterior, and superior directions closely parallels that of the adjacent nasal region, as described below. It is evident also that the floor of the orbit, as well as several other maxillary regions, does not move in a "downward and forward" course. Addition of bone on the inner but lateral-sloping floor of the orbital cavity does not decrease the size of the cavity itself. Rather, the entire orbital cavity is becoming relocated in anterior and lateral directions by these movements. The internal dimensions of the orbit are maintained in constant proportion, just as the wide ends of other $V$ - or funnel-shaped struetures move and simultaneously maintain their dimensions or increase in size.

The orbital surface of the maxilla (floor of the orbit) is composed of a thin single plate of lamellar bone. A middle zone of diploë is lacking. The endosteal surface is resorptive in character, and the periosteal side is depository (Fig. 8). This thin cortex also bounds the superior limit of the maxillary sinus. The cortical lining of this sinus was observed to be generally resorptive in nature, regardless of the particular part of the maxillary bone which covers it. This would suggest a generalized process of sinus enlargement during maxillary growth by resorptive removal from the inside combined with regional deposition on the various "outer" surfaces. As the growing sinus expands into areas of the maxilla which are already occupied by spongy bone, a slight compaction of marginal cancellous spaces takes place, thereby providing a thin cortical wall. This cortex continues to move during growth by a process of cancellous compaction combined with resorption from the opposite, inner side of the enlarging sinus.

The growth sequence of the adjacent zygomatic bone is coosdinated with the growth movements of the orbital surface. The anterior face of the zygomatic bone (area 16) and the entire cutaneous side of the lateral rim of the orbit are resorptive in nature. This arrangement contributes to the general posterior growth of the zygomatic bone. The medial surface of the frontal process of the zygomatic bone (area 18) is also resorptive (Fig. 6), thereby contributing to the movement of this area in a lateral as well as a posterior course. This direction of growth thus makes way for the laterally enlarging orbital surface (area 8 ) as it moves progressively into those areas previously oceupied by the moving lateral rim.

THE NASAL REGION. The lateral wall of the nasal cavity (frontal process of the maxilla, areas 9 and 10 ) is oriented in such a manner that it faces in the same general directions as the floor of the orbit-laterally, anteriorly, and superiorly. Appositional hone growth brings about simultaneous growth movements of this region in corresponding directions (Fig. 10). The inner or mucosal side of the frontal process (areas 13 and 15) has a resorptive periosteal surface (Figs. 4 and 5). Growth of the bony walls of the nose thus serves to enlarge 
the breadth of the nasal cavities, in conjunction with the moving orbital floor, and also to increase the size of the nose in both vertical and anterior dimensions. The adjacent nasal bones themselves also follow this same growth pattern. The outer facial surface of these bones (area 21) receives appositional deposits together with corresponding resorption from the contralateral inner (mucosal) surfaces (area 23).

Within the nasal eavity, the region of junction between the maxilla and the inferior concha is a prominent bony ridge (crista conchalis, area 14). Unlike the remainder of the cortical lining in the nasal cavity, this single localized area is composed of periosteal rather than endosteal compact bone.

VARIATIONS. Slight variations have been found in the exact placement of periosteal-to-endosteal contaets between the various numbered areas pictured in Fig. 1. Except for variations occurring at the line of contact between areas 1 and 5, they did not affect the basic patterns of periosteal-endosteal distributions diagrammed in these illustrations. In areas 1 and 5 , however, the line of periosteal-endosteal reversal was observed to vary within the relatively broad limits designated as area 3 in Fig. $1, B$ and by the symbol $T$ in Fig. 1, $D$. Variation in this particular region would affect the amount and location of lateral growth in the enlarging maxillary body and thereby serve to influence the actual width of the dental arch and the contour of its curvalure.

Variation was noted in area 17 of the zygomatic bone. In two of the specimens examined, this entire lateral surface was composed of endosteal bone rather than the more commonly observed periosteal cortex pictured in Fig. 2, C. The contralateral medial side (area 20 ) was correspondingly composed of periosteal bone rather than the more typical endosteal cortical bone tissue. The effect of this variation would be to limit the growth and movement of the zygomatic bone to an essentially posterior course with little increase in lateral dimensions, as previously described.

In most of the individual nasal bones studied, variable and quite small restricted patches of endosteal cortical bone were found at the free anteriorventral margin (area 22). In two specimens, however, there was a somewhat more extreme variation in which the cephalic two thirds of the external side of the nasal bone was composed of an inward-growing endosteal cortex. The opposite side, within the nasal cavity itself, showed a periosteal cortex. The inferior third of the bone demonstrated a converse pattern in which the external side was periosteal in origin whereas the internal surface was composed of endosteal bone. These combinations would serve to depress the bridge of the nose progressively while simultaneously enlarging and broadening the anterior free margin.

\section{DISCUSSION}

The maxillary body grows in an apparent forward and downward direction; yet actual increase in size is a result of new bone growth on posterior surfaces resulting in an extension of the dental arch. This paradoxical manner of growth parallels the mechanism of development in the mandible. The mandible, like the maxilla, is continuously repositioned in a forward direction as 
actual new growth proceds posteriorly. In understanding of this situation is complicated by observations in the present study that much of the anterior facial surface is actually losorptive in character. The face, therefore, is receding as it is simultancously being repositioned in an anterior direction.

It is evident that the generalization concerning "forward and downward movement produced by posterior growth" is in itself only partially accurate. Considering all of the various parts and areas of the entire maxila, with its many contours, ridges, depressions, and bony processes, the actual directions of regional growth necessarily proceed in a complex variety of divergent directions. It has been shown that growth in many adjacent areas proceeds in directions away from each other, so that continued growth has the effect of drawing out the dimensions of the whole bone. These growth increases make possible a lengthening of the dental arch itself and, at the same time, the proportionate growth of the orbit, nasal cavity, oral cavity, infratemporal fossa, maxillary sinuses, and all of the other various component parts of the maxillary bone. The principle of "area relocation" is a key consideration to the interpretation of these complex and multidirectional growth movements. As growth continues, specific local areas come to occupy new actual positions, in succession, as the entire bone enlarges. These growth shifts and changes involve corresponding and sequential remodeling adjustments in order to maintain the same shape, relative positions, and constant proportions of each individual area in the maxilla as a whole.

FORWARD REPOSITIONING OF THE MAXILLA DURING GROWTH. Whether the pacemaker of the various maxillary growth movements and of other facial components is a function of the nasal septum, sutures, brainease, periosteum, or progressive and interrelated combinations of all these is secondary to the particular objectives of the present study. As the maxilla grows, regardless of the particular nature and location of primary growth stimuli, the entire bone undergoes remodeling alterations and adjustments required as an essential and companion part of the growth process. For example, the movements involved in the progressive posterior relocation of the zygomatic process represent remodeling adjustments to the general process of maxillary growth. These remodeling movements of the zygomatic process, however, are not directly dependent upon the primary centers of maxillary growth. The situation is comparable to the growth of a long bone in that complex remodeling changes occurring in the metaphysis and diaphysis function separately from the activity of the primary growth center (epiphyseal plate) although dependent upon it as a pacemaker. ${ }^{3}$ The mandible, with a "growth center" in the condyle, presents a parallel situation. Extensive, sequential remodeling changes occur in all parts of the growing mandible, including the coronoid process, ramus, gonial angle, lingual tuberosity, and chin. These remodeling adjustments function as separate processes to endochondral bone growth activity occurring within the mandibular condyle. In the development of any bone, remodeling changes are a necessary consequence of growth but do not in themselves represent a primary growth stimulus for the bone as a whole. In the maxilla, its component parts receive extensive remodeling adjustments, and these movements are complementary to, but neces- 
sarily separate from, growth activity at the basic growth centers, including the nasal septum and sutures.

MESIAL DRIFT. Posterior growth of the young postnatal maxilla at its free ends provides a continuously elongating dental arch as the number of teeth increases. Over-all growth of the maxilla requires constant structural adjustment as growth movements produce the need for relocation of its various parts. In the same sense, a complementary process for the repositioning of teeth is also required. Because specific areas of tooth-bearing maxillary bone come to occupy, in sequence, different relative locations during successive stages of growth, teeth positioned in this bone must receive corresponding relocation in order to maintain constant relationships and positions. It has been assumed that during movements of teeth in maxillary or mandibular bone, the bone itself is relatively stable in position except for mesial and distal surfaces of alveolar walls. It is widely accepted that teeth "drift" through this relatively immobile and stable cortical bone by a process of resorption and apposition on inner alveolar surfaces. In the light of our present knowledge of maxillary and mandibular remodeling, an expansion of this concept is needed. Virtually all areas of the bone itself, as well as teeth, undergo continuous and extensive movements, shifts in location, and widespread drifting of all cortical plates in a variety of directions. Such movements of bone proceed simultaneously with tooth movements and undoubtedly contribute to them.

In Fig. 2, $A$, it is seen that the premaxillary area receives posterior relocation and thereby comes to occupy new levels relative to its former position. In succession, the incisor area continues to become sequentially relocated in its actual positions into more posterior locations which previously were occupied by different levels of the maxilla. It is apparent also that the incisor teeth must necessarily be carried with the moving bone in a corresponding manner and direction by a process of "drift." 'These various tooth movements and the bony adjustments adapted to them follow, in principle, the concept of the $V$. Since incisor areas are constantly being relocated into more medial positions during progressive backward growth, the continuous deposition of new bone on the inner side of the $V$ (the lingual surface of the entire maxillary arch), together with resorption from the outer surface (labial surfaces of the premaxilla), results in a shift of the various anterior parts of the maxillary arch sequentially into more medial positions toward the midline. It is not clear from the evidence at hand whether the teeth are moving in an actual mesial direction corresponding to the medial movement of the bone. It is conceivable, but not yet demonstrated, that they may drift in a compensatory distal direction in order to accommodate the medial direction of bone growth. Such distal drift, if it occurs at all, could be an actual movement or, perhaps, only an apparent drift involving a medial movement of the bone past teeth. This particular question should receive further detailed study. Teeth located in the general molar area of the maxillary arch are not subject to shifts in the same manner and the same directions as cuspids and incisors. As the whole maxilla is moved in an anterior direction, these teeth are carried in a like course, and corresponding bony adjustments may be required during the successive relocation of the various maxillary areas. These 
would involve familiar processes of appositional building on distal alveolar walls with complementary resorption from mesial walls.

The observations and interpretations just described indicate that tecth ale subjected to movements during growth in order to accommodate the general process of growth itself. The importance of considering facial growth in an interpretation of tooth movements was first suggested by Brodie, ${ }^{33}$ and evidence in the present study strongly supports such an approach. The occurrence of tooth migrations through bone appears to represent a physiologic mechanism adapted to the maintenance of constant relationships in position between tooth and specific areas of growing, moving bone. A secondary function is the maintenance of contact between adjacent teeth. Movement of the unerupted tooth germ has long been a puzzling problem, since some mechanical forces (mastication, tongue movements, etc.) are not yet a factor. The variety of maxillary growth movements described in the preceding paragraphs, however, may also represent a developmental basis for such tooth migrations. It is urged that detailed correlative studies between movements of tooth-bearing bone in relation to growth, remodeling, and corresponding tooth movements be undertaken.

\section{SUMMARY AND CONCLUSIONS}

As the maxilla increases in size, its various parts and regions come to occupy, in sequential order, new positions in the bone. This requires a mechanism of structural adjustment which brings about actual shifts in the location of specific parts in order to maintain constant shape and relative positions.

The postnatal growth of the human maxilla parallels that of the mandible in that forward and downward movement of the growing bone as a whole is a result of growth which takes place in a posterior direction with corresponding repositioning of the entire bone in a forward course. This growth pattern is one of several adaptations to the presence of teeth in the maxilla and mandible, and it makes possible elongation of the dental arch at its free (distal) ends. Such growth permits a progressive increase in the number of teeth which can take place only at the posterior ends of the dental arch. It also involves a complex series of corresponding remodeling changes in all of the various parts of both the maxilla and the mandible.

The generalization that the maxilla is thrust downward and forward by growth in posterior and superior parts of the bone is an oversimplification and, if not qualified, can lead to inaccurate assumptions. Growth does occur in this manner in certain specific areas, but it also proceeds in a complex variety of other directions in different major regions of the maxilla. The over-all size of the face increases by a series of specific growth movements in several individual areas which proceed away from each other, thereby drawing out the dimensions of the maxilla in several different directions.

Bone deposits are added along the posterior margin of the maxillary tuberosity. This functions to lengthen the dental arch and to enlarge the anteriorposterior dimensions of the entire maxillary body. Coordinated with this increase is the progressive movement of the entire zygomatic process in a corresponding posterior direction. This sequence serves to maintain continuously the constant 
position of the zygomatic process relative to the remainder of the maxilla. The separate zygomatic bone also moves in a posterior course by a combination of resorption from its anterior surfaces and deposition along its posterior side. The face simultaneously enlarges in breadth by proportionate bone apposition on the lateral surface of the zygomatic arch with corresponding resorption from its medial surface.

The floor of the orbit faecs superiorly, latcrally, and slightly anteriorly. Surface deposition results in growth proceeding in all three corresponding directions. Resorption from the lateral surface of the orbital rim functions to make way for the laterally moving orbital surface of the maxilla in the floor of the orbital cavity. The nasal area of the maxilla, together with its separate nasal bones, also faces in similar lateral, anterior, and superior directions. Growth proceeds in these same directions by surface bone deposition, thereby increasing the internal size of the nasal cavity by an elongation and expansion of its vertical and horizontal dimensions. The bony cortex lining the inner surface of the nasal cavity undergoes periosteal surface removal of bone as its endosteal side receives simultaneous deposits of new bone.

The palatine processes of the maxilla grow in a generally downward direction by a combination of surface deposition on the entire oral side of the palatal cortex with resorptive removal from the opposite nasal side as well as from periosteal labial surfaces of the anterior maxillary areh.

The premaxillary part of the maxilla grows in a downward direction. The surface orientation of this area is such that downward movement is brought about by resorptive removal from the periosteal surface of the labial cortex which faces away from the direction of growth. The endosteal side of its cortex and the periosteal surface of the lingual cortex receive new bone deposits. This growth pattern also produces a slight "recession" of the incisor area in a posterior direction, a situation also present in the human mandible.

It is suggested that the various remodeling movements of the growing maxillary bone contribute to a functional hasis for the drifting of teeth. Adjustments in the position of erupted as well as unerupted teeth appear to be required as a result of growth and remodeling movements of tooth-bearing bone.

It is also suggested that the variety of specific remodeling processes associated with maxillary and mandibular growth contributes to the characteristic age changes in the gross appearance of the human face.

This study was supported by United States Public Health Service Grant DE09103. The authors are grateful to Mr. William L. Brudon and Miss Lois Dirkse for providing the effective series of illustrations used in this report.

\section{REFERENCES}

1. Enlow, D. H.: A Study of the Post-natal Growth and Remodeling of Bone, Am. J. Anat. 110: 79-102, 1962 .

2. Enlow, D. H.: Functions of the Haversian System, Am. J. Anat. 110: 269-306, 1962.

3. Enlow, D. H.: Prineiples of Bone Remodeling, Springfield, Ill., 1963, Charles C Thomas, Publisher.

4. Enlow, D. H.: A Study of the Postnatal Growth of the Human Mandible, Am. J. OrthoDontics 50: 25-50, 1964. 
5. Gregory, W. K.: Our Face From Fish to Man, New York, 1929, G. P. Putnam's Sons.

6. Gregory, W. K.: Certain Critical Stages in the Evolution of the Vertebrate Taws, INT. I. ORTHODONTIA 17: 1138-1148, 1931.

7. Krogman, W. M.: The Problem of Growth Changes in Face and Skull as Viewed From a Comparative Study of Anthropoids and Man, Dental Cosmos 72: 624, 1930.

8. Weidenreich, Franz: Apes, Giants, and Man, Chicago, 1946, University of Chicago Press.

9. De Beer, G. R.: The Development of the Vertebrate Skull, London, 1937, Oxford University Press.

10. Dixon, Andrew D.: The Early Development of the Maxilla, D. Practitioner 3: 331-336, 1953.

11. Woo, J. K.: Ossification and Growth of the Human Maxilla, Premaxilla, and Palate Bones, Anat. Rec. 105: 731-761, 1949.

12. Baer, M. J.: Patterns of Growth of the Skull as Revealed by Vital Staining, Human Biol. 26: 80-126, 1954.

13. Brash, J. C.: The Growth of the Jaws and Palate, London, 1924, Dental Board of the United Kingdom, pp. 23-66.

14. Brash, J. C.: Some Problems in the Growth and Developmental Mechanics of Bone, Edinburgh M. J. 41: 305-387, 1934.

15. Hunter, John: Natural History of the Human Teeth, London, 1771, John Johnson.

16. Brodie, Allan G.: On the Growth Pattern of the Human Head, Am. J. Anat. 68: 209-262, 1941.

17. Brodie, Allan G.: Behavior of Normal and Abnormal Facial Growth Patterns, AM. J. ORTHODONTICS 27: 633-647, 1941.

18. Salzmann, J. A.: The Research Workshop on Cephalometrics, Am. J. Orthodontics 46: $834,1960$.

19. Krogman, W. M., and Sassouni, V.: A Syllabus in Roentgenographic Cephalometry, Philadelphia, 1957, Philadelphia Center for Research in Child Growth.

90. Moss, Melvin I.: Growth of the Calvaria in the Rat, Am. J. Anat. 94: 336-361, 1954.

21. Gans, B. J., and Sarnat, B. G.: Sutural Facial Growth of the Macaca Rhesus Monkey; A Gross and Serial Roentgenographic Study by Means of Metallic Implants, AM. J. ORTHODONTICS 37: 827-841, 1951.

22. Salzmann, J. A.: Prineiples of Orthodontics, ed. 2, Plitadelphia, 1950, J. B. Lippineott Company.

23. Weinmann, J. P., and Harry Sicher: Bone and Bones, ed. 2, St. Louis, 1955, The C. V. Mosby Company.

24. Moyers, R. E.: Handbook of Orthodonties, ed. 2, Chicago, 1963, Year Book Publishers, Inc.

25. Scott, James Henderson, and Symons, N. B. B.: Introduction to Dental Anatomy, ed. 2, Edinburgh, 1958, E. \& S. Livingstone, Ltd.

26. Massler, M., and Schour, I.: The Growth Pattern of the Cranial Vault in the Albino Rat as Measured by Vital Staining With Alizarine Red " $S$," Anat. Rec. 110: 83-101, 1941.

27. Pritchard, J. J., and Scott, J. H.: The Structure and Development of Cranial and Facial Sutures, .T. Anat. 90: 73-86, 1956.

28. Scott, James H.: Growth at the Facial Sutures, AM. J. ORThodontics 42: 381-387, 1956.

29. Seott, James H.: The Cartilage of the Nasal Septum, Brit. D. J. 95: 37-43, 1953.

30. Scott, James H.: Further Studies on the Growth of the Human Face, Proc. Roy. Soc. Med. 52: 263-268, 1958.

31. Scott, James H.: The Growth of the Human Face, Proc. Roy. Soe. Med. 47: 91-100, 1953.

32. Brodie, Allan G.: Jate Growth Changes in the Human Face, Angle Orthodontist 23: 146-157, 1953.

33. Brodie, Allan G.: Present Status of Knowledge Concerning Movement of the Tooth Germ Through the Jaw, J. Am. Dent. A. 21: 1830-1838, 1934.

34. Björk, Arne: Variations in the Growth Pattern of the Human Mandible: Longitudinal Radiographic Study by the Implant Method, J. D. Res. 42: 400-411, 1963. 